\title{
Asiaticoside, a component of Centella asiatica, inhibits melanogenesis in B16F10 mouse melanoma
}

\author{
KU JUNG KWON ${ }^{1}$, SEUNGHEE BAE ${ }^{1}$, KARAM KIM $^{1}$, IN SOOK AN ${ }^{1}$, \\ KYU JOONG AHN ${ }^{2}$, SUNGKWAN AN ${ }^{1}$ and HWA JUN CHA ${ }^{1}$ \\ ${ }^{1}$ Korea Institute for Skin and Clinical Sciences and Molecular-Targeted Drug Research Center, Konkuk University; \\ ${ }^{2}$ Department of Dermatology, Konkuk University School of Medicine, Seoul 143-701, Republic of Korea
}

Received August 20, 2013; Accepted March 7, 2014

DOI: $10.3892 / \mathrm{mmr} .2014 .2159$

\begin{abstract}
Melanogenesis is the process of generating pigmentation via melanin synthesis and delivery. Three key enzymes, tyrosinase, tyrosinase-related protein 1 (TRP1) and TRP2, metabolize melanin from L-tyrosine. Melanin synthesizing enzymes are regulated by microphthalmia-associated transcription factor (MITF). The titrated extract of Centella asiatica (TECA) contains the major components asiatic acid, asiaticoside and madecassic acid. The present study revealed that TECA reduces the melanin content in melanocytes. Moreover, the asiaticoside contained in TECA modulated melanogenesis by inhibiting tyrosinase mRNA expression. The decrease in tyrosinase mRNA levels was mediated through MITF. Uniquely, asiaticoside inhibited MITF by decreasing its DNA binding affinity. In conclusion, the results of the present study indicate that asiaticoside treatment may have beneficial effects in hyperpigmentation diseases or for skin whitening.
\end{abstract}

\section{Introduction}

Melanogenesis is the process of melanin synthesis and delivery through various enzymes (1). In particular, tyrosinase, tyrosinase-related protein 1 (TRP1), and tyrosinase related protein 2 (TRP2) are key enzymes in melanin synthesis (2,3). The melanin synthesizing enzymes transfer L-tyrosine to melanin though a multistep transformation (4). Microphthalmia-associated transcription factor (MITF) transcriptionally regulates these key enzymes in melanocytes (5). Therefore, the regulation of MITF is central for hyper- or

Correspondence to: Professor Sungkwan An or Dr Hwa Jun Cha, Korea Institute for Skin and Clinical Sciences and Molecular-Targeted Drug Research Center, Konkuk University, 120 Neungdong-ro, Gwangjin-gu, Seoul 143-701, Republic of Korea

E-mail: ansfgrc@konkuk.ac.kr

E-mail: hjcha@konkuk.ac.kr

Key words: microphthalmia-associated transcription factor, melanin, asiaticoside, Centella asiatica hypo-pigmentation diseases and for skin whitening for cosmetic purposes $(6,7)$.

Centella asiatica is a medicinal plant widely used in South Asia. C. asiatica extracts have therapeutic applications, particularly in neuroprotection and wound healing (8-11). In addition, its extracts are widely used for the treatment of inflammatory skin disorders, including leprosy, lupus, varicose ulcers, eczema, atopic dermatitis and psoriasis (12). The C. asiatica extract is mainly administered as a titrated extract. Titrated extract of $C$. asiatica (TECA) contains asiatic acid, asiaticoside and madecassic acid $(12,13)$. In dermatology, these components are used to achieve preventive and therapeutic effects. Asiaticoside prevents ultraviolet A-dependent photoaging by suppressing ultraviolet A-induced reactive oxygen species production (14). In addition, asiatic acid, madecassic acid and asiaticoside induce collagen I synthesis (15).

Recently, Saraf et al (16) indicated that a cosmetic formulation containing $C$. asiatica may possess de-melanogenic potential (16). However, the mechanism behind the activity of $C$. asiatica in melanocytes has yet to be elucidated. Therefore, a detailed understanding of the changes in melanogenesis is important for elucidating the mechanism of asiaticoside-dependent hypopigmentation. The present study aimed to determine how $C$. asiatica, particularly asiaticoside, affects melanogenesis.

\section{Materials and methods}

Materials and cell culture. TECA was obtained from Bayer (Leverkusen, Germany). Asiatic acid, madecassic acid, asiaticoside and 1-methyl-3-(2-methylpropyl)-7H-purine-2,6-dione (IBMX) were purchased from Sigma Aldrich (St. Louis, MO, USA). B16F10 cells were purchased from Korea Cell Line Bank (Seoul, Korea) and were cultured in Dulbecco's modified Eagle's medium (Sigma Aldrich) supplemented with $10 \%$ fetal bovine serum (Sigma Aldrich) and $1 \%$ penicillin-streptomycin $\left(\mathrm{Gibco}^{\circledR}\right.$, Life Technologies, Carlsbad, CA, USA). Cultures were maintained at $37^{\circ} \mathrm{C}$ with $5 \% \mathrm{CO}_{2}$.

Viability assays. The B16F10 cells were seeded in 96-well plates at a density of $4 \times 10^{3}$ cells per well. The cells were cultured with TECA, asiatic acid, madecassic acid or 
asiaticoside. After 24 or $48 \mathrm{~h}$, the cells were incubated with $0.5 \mathrm{mg} / \mathrm{ml}$ MTT for $2 \mathrm{~h}$. Formazan crystals were dissolved in dimethyl sulfoxide. The mean absorbance at $405 \mathrm{~nm}$ was assessed using an EL800 Absorbance Microplate Reader (Molecular Devices, Sunnyvale, CA, USA).

Assessment of melanin content. The melanin content was assessed using the method previously described by Hosoi et al with several modifications (17). The B16F10 cells were seeded at a density of $2 \times 10^{5}$ cells in $60-\mathrm{mm}$ culture dishes. The cells were cultured with TECA, asiatic acid, madecassic acid or asiaticoside. Following incubation, the melanocytes were washed in phosphate-buffered saline and lysed in $1 \mathrm{~N} \mathrm{NaOH}$. The mean absorbance at $405 \mathrm{~nm}$ was detected using an EL800 Absorbance Microplate Reader (Molecular Devices).

Western blot analysis. The B16F10 cells were cultured with TECA, asiatic acid, madecassic acid or asiaticoside. Following incubation, the cells were harvested and lysed in radioimmunoprecipitation assay buffer $[50 \mathrm{mM}$ Tris- $\mathrm{Cl}$ (pH 7.5), $150 \mathrm{mM} \mathrm{NaCl}, 1 \% \mathrm{NP}-40,0.5 \%$ deoxycholic acid and $0.1 \%$ sodium dodecyl sulfate]. The total protein concentration of the cell lysates was determined using a protein assay (Bio-Rad Laboratories, Hercules, CA, USA). Equal amounts of protein were separated with 12 or $10 \%$ SDS-PAGE and transferred to nitrocellulose membranes (Whatman, Dassel, Germany). The membrane was blocked in 5\% skimmed milk. Anti-tyrosinase goat polyclonal IgG, anti-TRP1 goat polyclonal $\operatorname{IgG}$, anti-MITF rabbit monoclonal $\operatorname{IgG}$ and anti- $\beta$-actin mouse monoclonal IgG antibodies were used to detect specific proteins and were purchased from Santa Cruz Biotechnology, Inc. (Santa Cruz, CA, USA). The membranes were visualized using SuperSignal West Pico (Pierce Biotechnology, Inc., Rockford, IL, USA) and Las-4000 (Fujifilm, Minato-ku, Japan).

Quantitative polymerase chain reaction $(q P C R)$. Total RNA from the B16F10 cells was isolated using RiboEX (Geneall, Seoul, Korea) according to the manufacturer's instructions. cDNA was synthesized using reverse transcriptase (Bioneer Corp., Daejeon, Korea) and used as template for the PCR. The PCR mixtures contained template, $0.5 \mu \mathrm{M}$ primers and HOT FIREPol Eva Green ${ }^{\circledR}$ qPCR Mix Plus (Solis Biodyne, Tartu, Estonia) from a SYBR green I-based qPCR kit. Reactions were run in LinegeneK (BioER, Hangzhou, China) using the following program: $5 \mathrm{~min}$ denaturation at $95^{\circ} \mathrm{C}$, followed by 40 cycles of $15 \mathrm{sec}$ denaturation at $95^{\circ} \mathrm{C}, 15 \mathrm{sec}$ annealing at $58^{\circ} \mathrm{C}$ and $15 \mathrm{sec}$ polymerization at $72^{\circ} \mathrm{C}$. The sequences of the primers used were as follows: Tyrosinase forward, 5'-ACACACTGGAAGGATTTGCC-3' and reverse, 5'-GAGCGGTATGAAAGGAACCA-3'; and $\beta$-actin forward, 5'-CGACAGGATGCAGAAGGAG-3' and reverse, 5'-ACATCTGCTGGAAGGTGGA-3'. The relative quantity of all samples was calculated using a serial dilution standard curve.

Chromatin immunoprecipitation $($ ChIP). Following treatment with TECA and TECA components, the cells were washed in ice-cold phosphate-buffered saline and cross-linked for
A

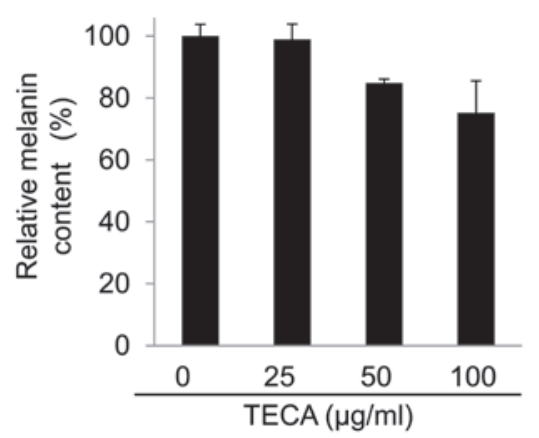

B

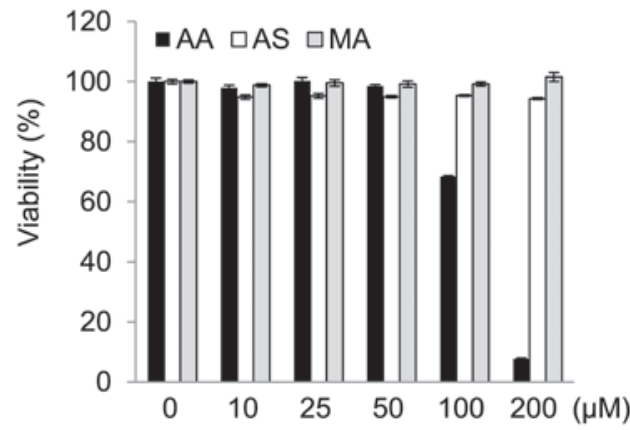

C

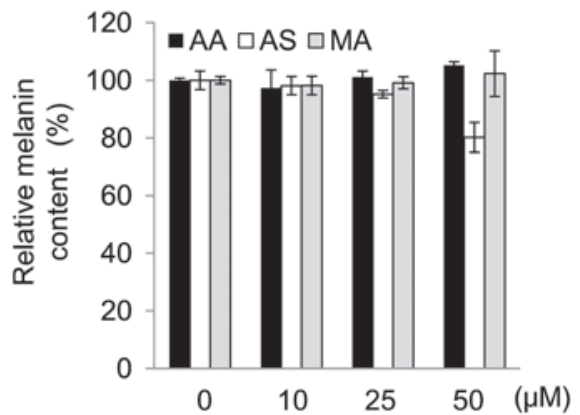

Figure 1. Inhibitory effect of TECA and TECA components in melanogenesis. B16F10 cells were incubated with the indicated concentrations of TECA or TECA components (asiatic acid, asiaticoside and madecassic acid). (A) The melanin content of TECA-treated B16F10 cells was assessed via the optical density $(405 \mathrm{~nm})$. Optical density results are expressed as the relative difference from the control. (B) B16F10 cells were cultured with the indicated concentrations of asiatic acid, asiaticoside and madecassic acid for $48 \mathrm{~h}$. Cell viability was measured using MTT assays. Cytotoxicity was calculated using untreated B16F10 cells as a control. Data are expressed as the mean \pm standard deviation from three experiments. (C) Following $48 \mathrm{~h}$ of incubation, cell lysates were analyzed by measuring the optical density at $405 \mathrm{~nm}$. Data are presented as the mean \pm standard deviation from three experiments. TECA, titrated extract of Centella asiatica; AA, asiatic acid; AS, asiaticoside; MA, madecassic acid.

15 min at $25^{\circ} \mathrm{C}$ with $1 \%$ formaldehyde. The cross-linked cells were harvested and resuspended in ChIP lysis buffer [50 mM HEPES (pH 7.5), $140 \mathrm{mM} \mathrm{NaCl}, 1 \%$ Triton X100]. The cells were sonicated and immunoprecipitated with the anti-MITF antibody (Santa Cruz Biotechnology, Inc.). Following immunoprecipitation, cross-linked proteins were degraded using protease $\mathrm{K}$, and DNA was collected using the PCR purification kit (Geneall). The tyrosinase promoter site was detected using PCR. The following primer sequences for the tyrosinase promoter were used: Forward, 5'-AGTCATGTGCTTTGCAGAAGAT-3' and reverse, 5'-CAGCCAAGAACATTTTCTCCTT-3'. 

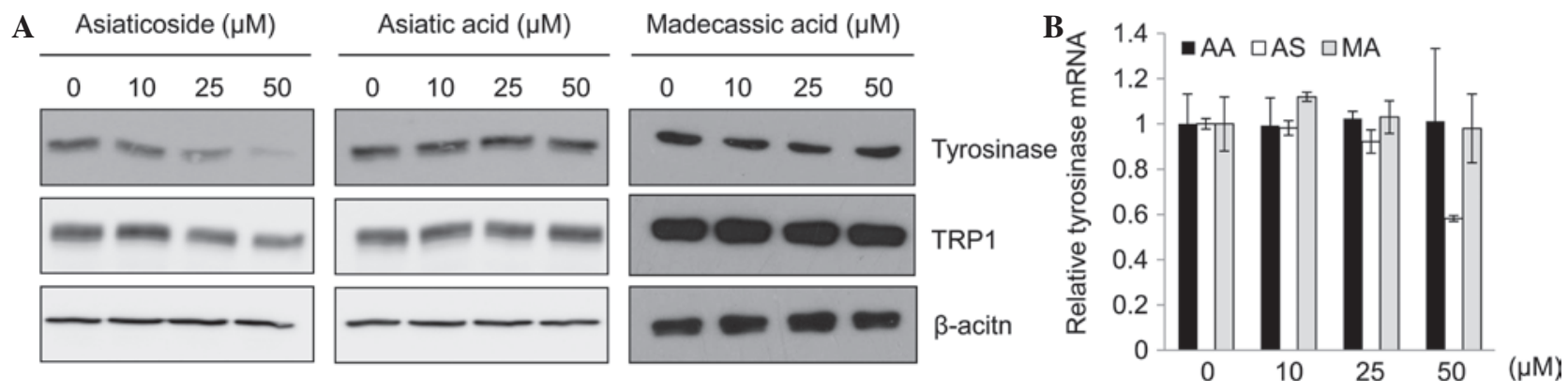

Figure 2. Effect of asiaticoside on tyrosinase expression. B16F10 cells were incubated with the indicated concentrations of TECA or TECA components (asiatic acid, asiaticoside and madecassic acid). (A) Following treatment with asiatic acid, asiaticoside and madecassic acid, the expression levels of tyrosinase, TRP1, and TRP2 were analyzed using SYBR Green I-based quantitative polymerase chain reaction (qPCR) assays. (B) The protein levels of tyrosinase were analyzed by western blot analysis. $\beta$-actin was used as a loading control. TECA, titrated extract of Centella asiatica; AA, asiatic acid; AS, asiaticoside; MA, madecassic acid; TRP, tyrosinase-related protein.

A

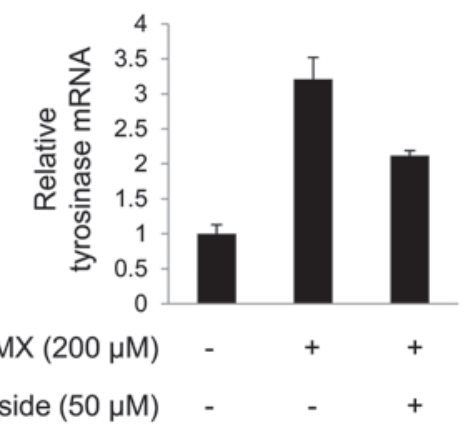

B

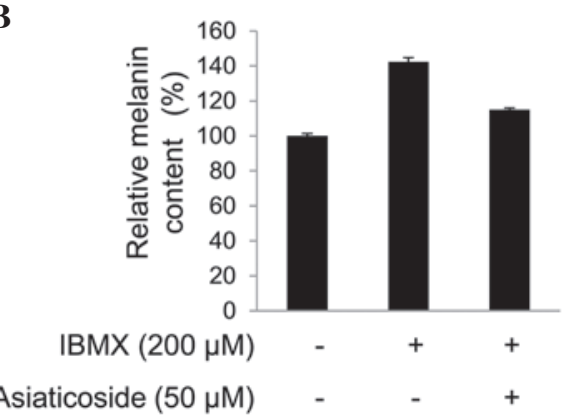

Figure 3. Effect of asiaticoside on IBMX-treated B16F10 cells. (A) B16F10 cells were incubated with the indicated concentrations of asiaticoside and IBMX. Following treatment, the expression levels of tyrosinase were analyzed using SYBR Green I-based quantitative polymerase chain reaction (qPCR). (B) The melanin content of the asiticoside-treated B16F10 cells was assessed via the optical density $(405 \mathrm{~nm})$. Optical density results are expressed as the relative difference from the control. IBMX, 1-methyl-3-(2-methylpropyl)-7H-purine-2,6-dione.

\section{Results}

TECA represses melanogenesis in B16F10 cells. To examine whether TECA possesses cytotoxic activity, the B16F10 cells were treated with TECA at various concentrations $(0,10,25,50$, 100 and $200 \mu \mathrm{g} / \mathrm{ml}$ ) for $48 \mathrm{~h}$. After $48 \mathrm{~h}$, no significant changes were observed in B16F10 cell viability with 10 to $100 \mu \mathrm{g} / \mathrm{ml}$ TECA (data not shown). Therefore, $<100 \mu \mathrm{g} / \mathrm{ml}$ TECA was used in the following experiments. As shown in Fig. 1A, TECA decreased the melanin content in the B16F10 cells in a concentration-dependent manner.
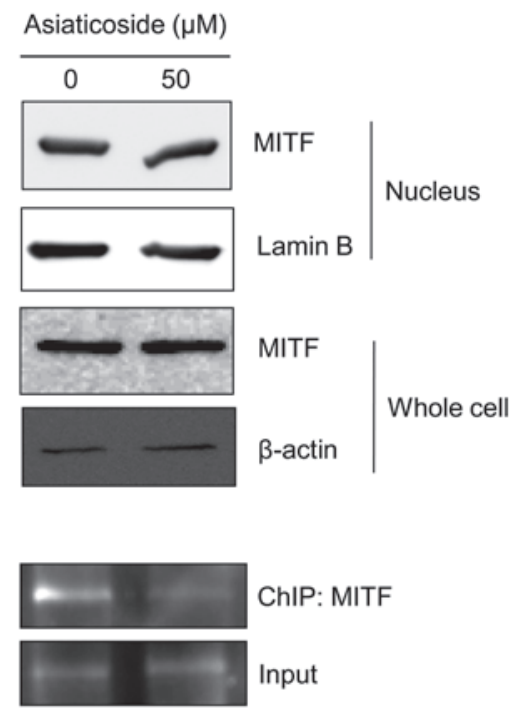

Figure 4. Inhibition of MITF activity in B16F10 cells incubated with asiaticoside at the indicated concentrations. Following treatment, the cells were divided into nuclear and cytosolic fractions. MITF protein levels were assessed using western blot analysis (top panel). Lamin B and $\beta$-actin were used as a loading control and a fraction positive control, respectively. The binding affinity and interaction between the tyrosinase promoter and MITF was assessed using ChIP (bottom panel). MITF, microphthalmia-associated transcription factor; ChIP, chromatin immunoprecipitation.

Asiaticoside, the major component of TECA, represses melanogenesis in B16F10 cells. As melanogenesis was repressed by TECA, the present study also investigated which of its components contributed to the repression of this process. The cytotoxic effects of the major components of TECA (asiaticoside, asiatic acid and madecassic acid) were assessed in the B16F10 cells using MTT assays. The dose-response curves to the TECA components in the B16F10 cells are shown in Fig. 1. Asiaticoside and madecassic acid $(0-200 \mu \mathrm{M})$ had no effect on cell viability (Fig. 1B). However, asiatic acid induced cytotoxicity at concentrations of $>100 \mu \mathrm{M}$ in the B16F10 cells. Therefore, the melanin content was assessed following incubation with $50 \mu \mathrm{M}$ asiatic acid. Under non-toxic conditions, the melanin content was decreased by asiaticoside, but not by asiatic acid or madecassic acid (Fig. 1C). As tyrosinase has a significant role in melanogenesis, the effects of asiaticoside on tyrosinase protein and mRNA expression 
in the B16F10 cells were assessed. Fig. 2A and B shows that asiaticoside suppressed tyrosinase mRNA and protein expression in the B16F10 cells.

In addition, asiaticoside repressed the melanogenesis induced by IBMX by reducing the level of tyrosinase mRNA (Fig. 3A). Consistent with this, the melanin content was decreased by asiaticoside in the B16F10 cells undergoing IBMX-induced melanogenesis (Fig. 3B). The decrease in tyrosinase mRNA is common in MITF-repressed melanocytes, as MITF transcriptionally regulates tyrosinase (5). Furthermore, IBMX, a cyclic adenosine monophosphate (cAMP) phosphodiesterase inhibitor, induces the level of expression in MITF target genes by increasing the level of cAMP (18). Therefore, MITF activity was assessed in the present study.

Asiaticoside decreases tyrosinase $m R N A$ expression by repressing the DNA binding affinity of MITF. As described in the introduction, MITF is a key regulator of melanogenesis and a possible target for the treatment of hyperpigmentation diseases $(6,7)$. Therefore, in the present study, it was hypothesized that the asiaticoside-dependent inhibition of melanogenesis could be regulated by MITF transactivation activity. To determine whether asiaticoside regulates MITF, the translocation and protein expression of MITF in asiaticoside-treated B16F10 cells was monitored. Asiaticoside did not regulate MITF expression in the whole cell lysates. In addition, the nuclear translocation of MITF was unchanged following incubation with asiaticoside. However, asiaticoside treatment altered the DNA binding affinity of MITF in the B16F10 cells (Fig. 4).

\section{Discussion}

C. asiatica extracts are used for treating psoriasis and wounds of the skin $(19,20)$. C. asiatica extracts promote wound healing through production of collagen (21). In a previous study, Saraf et al (16) reported that hydroalcoholic extracts of Curcuma caesia (rhizome), Areca catechu (seeds), Centella asiatica (leaves), Cinnamon zeylanicum (dried bark), and Tamarindus indica (fruit pulp) reduce melanin content, as measured by mexameter. However, the study indicated that herbal extracts enhance photoprotection by increasing the sun protection factor value (16). Therefore, the present study evaluated the effects of $C$. asiatica extracts on melanogenesis in melanocytes.

As shown in Fig. 1, TECA decreased the melanin content in the B16F10 cells in a concentration-dependent manner. Melanin is produced by a multi-step metabolic conversion of L-tyrosine to melanin (22). The metabolic synthesis of melanin is performed by enzymes such as tyrosinase, TRP1 and TRP2 (23). Tyrosinase is the major enzyme involved in the melanin metabolic pathway (23). In the present study, asiaticoside inhibited melanogenesis by the inhibition of tyrosinase protein and mRNA expression (Fig. 2A and B). In IBMX-induced melanogenesis, tyrosinase transcription is regulated by MITF (24). Therefore, to determine the effect of asiaticoside on MITF, the expression levels, translocation and DNA binding affinity of MITF protein were assessed in the present study. Notably, as shown Fig. 4, asiaticoside inhibits melanogenesis by interfering with the DNA binding of MITF. Similarly, Um et al (25) reported that \{1-[2-(4-chloro-phenoxy)-ethyl]-1H-benzoimidazol-2-ylsulfanyl $\}$-acetic acid inhibits melanogenesis by interfering with direct MITF binding to E-box DNA. In addition, MITF DNA binding is inhibited by PIAS3, a protein inhibitor of activated signal transduction and activator of transcription 3 (26).

In conclusion, these results indicate that TECA, and in particular, asiaticoside, inhibited melanogenesis by regulating the DNA binding affinity of MITF. Furthermore, the melanin content was reduced by decreasing the level of expression in tyrosinase and MITF target genes. Therefore, the present study revealed that asiaticoside is a novel candidate for melanogenesis inhibition through repression of DNA binding to MITF.

\section{Acknowledgements}

The present study was supported by the Konkuk University research support program.

\section{References}

1. Park HY, Kosmadaki M, Yaar M and Gilchrest BA: Cellular mechanisms regulating human melanogenesis. Cell Mol Life Sci 66: 1493-1506, 2009.

2. Tsukamoto K, Jackson IJ, Urabe K, Montague PM and Hearing VJ: A second tyrosinase-related protein, TRP-2, is a melanogenic enzyme termed DOPAchrome tautomerase. EMBO J 11: 519-526, 1992.

3. Negroiu G, Dwek RA and Petrescu SM: Tyrosinase-related protein-2 and -1 are trafficked on distinct routes in B16 melanoma cells. Biochem Biophys Res Commun 328: 914-921, 2005.

4. Slominski A, Tobin DJ, Shibahara S and Wortsman J: Melanin pigmentation in mammalian skin and its hormonal regulation. Physiol Rev 84: 1155-1228, 2004.

5. Vachtenheim J and Borovanský J: 'Transcription physiology' of pigment formation in melanocytes: central role of MITF. Exp Dermatol 19: 617-627, 2010.

6. Wan P, Hu Y and He L: Regulation of melanocyte pivotal transcription factor MITF by some other transcription factors. Mol Cell Biochem 354: 241-246, 2011.

7. Yi X, Zhao G, Zhang H, et al: MITF-siRNA formulation is a safe and effective therapy for human melasma. Mol Ther 19: 362-371, 2011.

8. MacKay D and Miller AL: Nutritional support for wound healing. Altern Med Rev 8: 359-377, 2003.

9. Brinkhaus B, Lindner M, Schuppan D and Hahn EG: Chemical, pharmacological and clinical profile of the East Asian medical plant Centella asiatica. Phytomedicine 7: 427-448, 2000.

10. da Rocha MD, Viegas FP, Campos HC, Nicastro PC, Fossaluzza PC, Fraga CA, Barreiro EJ and Viegas C Jr: The role of natural products in the discovery of new drug candidates for the treatment of neurodegenerative disorders II: Alzheimer's disease. CNS Neurol Disord Drug Targets 10: 251-270, 2011.

11. Defillipo PP, Raposo AH, Fedoce AG, Ferreira AS, Polonini HC, Gattaz WF and Raposo NR: Inhibition of cPLA2 and SPLA2 activities in primary cultures of rat cortical neurons by Centella asiatica water extract. Nat Prod Commun 7: 841-843, 2012.

12. Belcaro G, Maquart FX, Scoccianti M, Dugall M, Hosoi M, Cesarone MR, Luzzi R, Cornelli U, Ledda A and Feragalli B: TECA (Titrated Extract of Centella asiatica): new microcirculatory, biomolecular, and vascular application in preventive and clinical medicine. A status paper. Panminerva Med 53 (3 Suppl 1): 105-118, 2011.

13. Gohil KJ, Patel JA and Gajjar AK: Pharmacological review on Centella asiatica: a potential herbal cure-all. Indian J Pharm Sci 72: 546-556, 2010. 
14. Soo Lee Y, Jin DQ, Beak SM, Lee ES and Kim JA: Inhibition of ultraviolet-A-modulated signaling pathways by asiatic acid and ursolic acid in HaCaT human keratinocytes. Eur J Pharmacol 476: $173-178,2003$.

15. Bonte F, Dumas M, Chaudagne C and Meybeck A: Influence of asiatic acid, madecassic acid, and asiaticoside on human collagen I synthesis. Planta Med 60: 133-135, 1994.

16. Saraf S, Chhabra SK, Kaur CD and Saraf S: Development of photochemoprotective herbs containing cosmetic formulations for improving skin properties. J Cosmet Sci 63: 119-131, 2012.

17. Hosoi J, Abe E, Suda T and Kuroki T: Regulation of melanin synthesis of B16 mouse melanoma cells by 1 alpha, 25-dihydroxyvitamin D3 and retinoic acid. Cancer Res 45: 1474-1478, 1985.

18. Essayan DM: Cyclic nucleotide phosphodiesterases. J Allergy Clin Immunol 108: 671-680, 2001

19. Tenni R, Zanaboni G, De Agostini MP, Rossi A, Bendotti C and Cetta G: Effect of the triterpenoid fraction of Centella asiatica on macromolecules of the connective matrix in human skin fibroblast cultures. Ital J Biochem 37: 69-77, 1988.

20. Maquart FX, Chastang F, Simeon A, Birembaut P, Gillery P and Wegrowski Y: Triterpenes from Centella asiatica stimulate extracellular matrix accumulation in rat experimental wounds. Eur J Dermatol 9: 289-296, 1999.
21. Suguna L, Sivakumar P and Chandrakasan G: Effects of Centella asiatica extract on dermal wound healing in rats. Indian J Exp Biol 34: 1208-1211, 1996.

22. Letellier S, Garnier JP, Spy J and Bousquet B: Determination of the L-DOPA/L-tyrosine ratio in human plasma by high-performance liquid chromatography. Usefulness as a marker in metastatic malignant melanoma. J Chromatogr B Biomed Sci Appl 696: 9-17, 1997.

23. Ando H, Kondoh H, Ichihashi $\mathrm{M}$ and Hearing VJ: Approaches to identify inhibitors of melanin biosynthesis via the quality control of tyrosinase. J Invest Dermatol 127: 751-761, 2007.

24. Park SY, Jin ML, Kim YH, Kim Y and Lee SJ: Aromatic-turmerone inhibits $\alpha$-MSH and IBMX-induced melanogenesis by inactivating CREB and MITF signaling pathways. Arch Dermatol Res 303: 737-744, 2011.

25. Um JM, Kim HJ, Lee Y, Choi CH, Hoang Nguyen D, Lee HB, Shin JH, Tai No K and Kim EK: A small molecule inhibitor of Mitf-E-box DNA binding and its depigmenting effect in melan-a cells. J Eur Acad Dermatol Venereol 26: 1291-1297, 2012.

26. Levy C, Nechushtan H and Razin E: A new role for the STAT3 inhibitor, PIAS3: a repressor of microphthalmia transcription factor. J Biol Chem 277: 1962-1966, 2012. 